\title{
SEGURANÇA COM CONTROLE DE ENGENHARIA E ADESÃO À PRECAUÇÃO PADRÃO NO CATETERISMO VENOSO PERIFÉRICO: ESTUDO OBSERVACIONAL
}

\author{
SAFETY WITH ENGINEERING CONTROL \\ AND TREATMENT TO STANDARD PRECAUTION \\ IN PERIPHERAL VENOUS CATHETERIZATION: \\ OBSERVATIONAL STUDY
}

\section{SEGURIDAD CON CONTROL DE INGENIERÍA Y TRATAMIENTO SEGÚN PRECAUCIÓN ESTÁNDAR EN CATETERISMO VENOSO PERIFÉRICO: ESTUDIO OBSERVACIONAL}

\author{
Guilherme Nascimento de Azevedo ${ }^{1}$ \\ Damiana Aparecida Trindade Monteiro ${ }^{2}$ \\ Letícia Nunes Torres ${ }^{3}$ \\ Lúbia Alves dos Santos ${ }^{4}$ \\ Elucir Gir \\ Silmara Elaine Malaguti Toffano ${ }^{6}$
}

\begin{abstract}
Como citar este artigo: Azevedo GN, Monteiro DAT, Torres LN, Santos LA, Gir E, Toffano SEM. Segurança com controle de engenharia e adesão à precaução padrão no cateterismo venoso periférico: estudo observacional. Rev baiana enferm. 2022;36:e45618.

Objetivo: analisar o manuseio do dispositivo de segurança com controle de engenharia em cateter venoso periférico e adesão às Precauções Padrão por profissionais de enfermagem durante a punção venosa periférica em adultos. Método: observacional, descritivo, de abordagem quantitativa, realizado em unidade de internação clínica cirúrgica. Dados coletados por meio de instrumento semiestruturado, contendo variáveis referentes ao cateter e a observação do procedimento. Os dados foram analisados mediante estatística descritiva. Resultados: a amostra do estudo foi composta por 28 profissionais, sendo observados 235 procedimentos. A higienização das mãos com antisséptico foi realizada em 23\% (54); solução alcoólica em 10,6\% (25). Luvas de procedimento e óculos de proteção foram utilizados em 56,6\% (133) e 2,1\% (5), respectivamente. O cateter com dispositivo retrátil foi acionado incorretamente em 45,1\% (106). Conclusão: a maioria dos profissionais utilizou o cateter venoso periférico com controle de engenharia de maneira inadequada e a adesão às precauções padrão foi baixa.
\end{abstract}

\footnotetext{
Enfermeiro. Residente em Urgência e Trauma na Universidade Federal do Triângulo Mineiro. Uberaba, Minas Gerais, Brasil. guilhermenascimentoazevedo@hotmail.com. http://orcid.org/0000-0003-4012-8263.

Enfermeira. Mestre em Enfermagem. Universidade Federal do Triângulo Mineiro. Uberaba, Minas Gerais, Brasil. http://orcid.org/0000-0002-6740-7687.

Acadêmica de Enfermagem. Universidade Federal do Triângulo Mineiro. Uberaba, Minas Gerais, Brasil. http://orcid.org/0000-000 I-5654-234I .

Enfermeira. Residente em Urgência e Trauma na Universidade Federal do Triângulo Mineiro. Uberaba, Minas Gerais, Brasil. http://orcid.org/0000-0003-0467-876X.

Enfermeira. Doutora em Enfermagem. Professora Titular na Escola de Enfermagem de Ribeirão Preto da Universidade de São Paulo. Ribeirão Preto, São Paulo, Brasil. http://orcid.org/0000-0002-3757-4900.

6 Enfermeira. Doutora em Enfermagem. Professora Adjunta na Universidade Federal do Triângulo Mineiro. Uberaba, Minas Gerais, Brasil. http://orcid.org/0000-00029080-9123.
} 
Descritores: Cateterismo Periférico. Equipamentos de Proteção. Enfermagem do Trabalho. Equipamento de Proteção Individual. Estudo Observacional.

Objective: to analyze the handling of the safety device with engineering control in peripheral venous catheter and the adherence to Standard Precautions by nursing professionals during peripheral venous puncture in adults. Method: observational, descriptive study, with quantitative approach, performed in a surgical clinical hospitalization unit. Data collected through a semi-structured instrument, containing variables related to the catheter and the observation of the procedure. The data were analyzed using descriptive statistics. Results: the study sample consisted of 28 professionals, and 235 procedures were observed. Hand bygiene with antiseptic was performed in 23\% (54); alcohol solution in 10.6\% (25). Procedure gloves and goggles were used in 56.6\% (133) and 2.1\% (5), respectively. The catheter with retractable device was incorrectly activated in 45.1\% (106). Conclusion: most professionals used the peripheral venous catheter with inadequate engineering control and the standard precautions were low.

Descriptors: Peripheral Catheterization. Protective Equipment. Occupational Nursing. Personal Protective Equipment. Observational Study.

Objetivo: analizar el manejo del dispositivo de seguridad con control de ingeniería en catéter venoso periférico y el seguimiento de las precauciones estándar por parte de los profesionales de enfermería durante la punción venosa periférica en adultos. Método: abordaje observacional, descriptivo, cuantitativo, realizado en una unidad de hospitalización clínica quirúrgica. Datos recogidos a través de un instrumento semiestructurado, que contiene variables relacionadas con el catéter y la observación del procedimiento. Los datos fueron analizados mediante estadística descriptiva. Resultados: la muestra del estudio estuvo constituida por 28 profesionales, y se observaron 235 procedimientos. La higiene de manos con antiséptico se realizó en el 23\% (54); solución de alcohol en 10,6\% (25). Se utilizaron guantes y gafas de procedimiento en el 56,6\% (133) y el 2,1\% (5), respectivamente. El catéter con dispositivo retráctil se activó incorrectamente en el 45,1\% (106). Conclusión: la mayoría de los profesionales utilizaron el catéter venoso periférico con un control de ingeniería inadecuado y las precauciones estándar fueron bajas.

Descriptores: Cateterismo Periférico. Equipo de Protección. Enfermería Ocupacional. Equipo de Protección Personal. Estudio Observacional.

\section{Introdução}

O cateter venoso periférico (CVP) é um dispositivo utilizado rotineiramente nas instituições de saúde para infusão de medicamentos e soluções endovenosas, sendo um importante recurso terapêutico. A equipe de enfermagem é responsável pelo manuseio desses cateteres durante sua inserção, manutenção e cuidados após a terapia infunsional ${ }^{(1)}$.

Sendo uma prática frequente, torna-se imprescindivel que os profissionais de enfermagem sejam capacitados e tenham conhecimento científico quanto à escolha e o manejo do cateter, levando em consideração o tempo previsto de duração do tratamento, condições da rede venosa periférica, custo e benefícios ${ }^{(2)}$.

O CVP é o dispositivo invasivo mais utilizado no ambiente hospitalar. Estima-se que mais de 300 milhões de CVP sejam vendidos por ano nos Estados Unidos, e que, pelo menos, 80\% de todos os pacientes hospitalizados recebem durante sua internação algum tipo de terapia intravenosa ${ }^{(3)}$. Em um estudo realizado na Espanha estimou-se que, em média, 50\% dos pacientes internados receberam um tipo de cateter, dos quais $95 \%$ foram periféricos ${ }^{(4)}$.

A equipe de enfermagem dentro do ambiente hospitalar é responsável pela maior parte da realização de procedimentos invasivos, tornando-se vulnerável aos riscos ocupacionais, incluindo o biológico, em decorrência de reencape da agulha, descarte e transporte inadequado e contato com fluidos corporais ${ }^{(5-6)}$. O reencape da agulha por parte dos profissionais ainda é um motivo que acarreta acidente com perfurocortante ${ }^{(7)}$.

Para diminuição dos acidentes com perfurocortantes relacionados ao procedimento de punção venosa periférica (PVP), é recomendado o uso das Precauções Padrão (PP) associadas 
aos dispositivos de segurança com controle de engenharia (DSCE) $)^{(8-9)}$. Dados de pesquisa americana, realizada em 85 hospitais, apontaram redução dos acidentes em 38\% após o uso do $\operatorname{DSCE}^{(10)}$. Cateteres convencionais que não possuem dispositivo de segurança continuam a causar a maioria dos acidentes com agulha. Segundo estudo realizado em um hospital na Itália, eles foram responsáveis por $82 \%$ do total de acidentes ${ }^{(11)}$.

Não obstante a proteção contra a picada da agulha, ainda há a possibilidade de respingo de sangue no momento de ativação do dispositivo em determinados modelos de $\mathrm{CVP}^{(12)}$. O DSCE é classificado em duas categorias: os que são ativados manualmente e os que permitem a proteção passivamente no momento da retirada da agulha da cânula ${ }^{(13)}$.

No Brasil, é considerado como risco biológico uma exposição ocupacional a agentes biológicos, como microrganismos, toxinas, bactérias, fungos, entre outros ${ }^{(14)}$. Assim, o DSCE deve ser integrado ao CVP com tecnologia capaz de reduzir o risco de acidente, seja qual for o mecanismo de ativação ${ }^{(15)}$.

Devido à escassez de produções científicas acerca da temática, o presente estudo teve como objetivo analisar o manuseio do dispositivo de segurança com controle de engenharia em cateter venoso periférico e adesão às Precauções Padrão por profissionais de enfermagem durante a punção venosa periférica em adultos.

\section{Método}

Trata-se de estudo observacional, descritivo e de abordagem quantitativa, norteado pela ferramenta Strengthening the Reporting of Observational Studies in Epidemiology (STROBE). Realizado em um hospital público de ensino e de alta complexidade, localizado no interior de Minas Gerais e que atende a usuários do Sistema Único de Saúde (SUS). A coleta de dados foi realizada no período de fevereiro de 2018 a agosto de 2018 por pesquisadores previamente capacitados e alinhados quanto ao método de registro da observação.

A população foi composta por 28 profissionais da equipe de enfermagem (enfermeiros, técnicos e auxiliares de enfermagem) que atuavam na Unidade de Internação Clínica Cirúrgica. Consideraram-se como critérios de inclusão: pertencer à equipe de enfermagem na unidade em estudo e realizar a punção venosa periférica (PVP) no período de observação da coleta de dados. Foram excluídos os profissionais que desempenhavam apenas atividades administrativas.

Para a coleta de dados foram utilizados dois instrumentos: um contemplava as variáveis referentes ao profissional (categoria, sexo, idade e turno de trabalho) e outro, para o registro da observação da PVP (higiene das mãos, adesão às precauções padrão, tipo do dispositivo, momento para acionamento do dispositivo de segurança, contato com sangue, transporte do cateter venoso periférico, descarte do dispositivo logo após o procedimento). O instrumento foi validado quanto à forma e conteúdo por sete enfermeiros, quatro doutores e três mestres com expertise na temática.

Os pesquisadores observaram os profissionais durante o procedimento, diariamente, em todos os turnos de trabalho. As oportunidades de PVP observadas foram acompanhadas do momento da inserção até o descarte do material, sendo realizado pelos pesquisadores de forma a não comprometer a rotina assistencial e sem interferências externas.

As variáveis do instrumento foram codificadas e catalogadas em um banco de dados, duplamente digitados e validados no software Microsoft Office ${ }^{\circledR}$ do Excel ${ }^{\circledR}$. Posteriormente, os dados foram exportados e analisados descritivamente no software IBM SPSS ${ }^{\circledR}$, versão 21.0. Os dados foram analisados mediante estatística descritiva (frequências absolutas e porcentagens).

Foram consideradas as diretrizes e normas regulamentadoras de pesquisas envolvendo seres humanos, Resolução no 466/2012, do Conselho Nacional de Saúde, em todos os aspectos. 
O projeto de pesquisa foi aprovado pelo Comitê de Ética em Pesquisa (CEP) da Universidade Federal do Triângulo Mineiro sob n⿳⺈ 2.382.208. Os profissionais foram convidados a participar da pesquisa durante o turno de trabalho (matutino, vespertino e noturno), após a compreensão do Termo de Consentimento Livre e Esclarecido (TCLE) e informado os objetivos e a finalidade da pesquisa. Em casos de acidente ocupacional após a observação foi pontuado ao profissional a importância do registro da notificação e análise do acidente.

\section{Resultados}

A amostra do estudo foi composta por 28 profissionais de enfermagem, sendo $24(85,7 \%)$ técnicos e auxiliares de enfermagem e $4(14,3 \%)$ enfermeiros. A maior proporção foi do sexo feminino, 21 (75\%), com idade entre 23 e 67 anos e com experiência profissional entre 2 e 45 anos. Com relação ao turno de trabalho, o período noturno teve maior participação, 13 (46,4\%) (Tabela 1).

Tabela 1 - Perfil dos profissionais de enfermagem de um hospital público brasileiro, segundo categoria, sexo, faixa etária e turno de trabalho. Uberaba, Minas Gerais, Brasil - 2018. N=28

\begin{tabular}{|c|c|c|}
\hline Variável & $\mathbf{n}$ & $\%$ \\
\hline \multicolumn{3}{|l|}{ Categoria Profissional } \\
\hline Técnico e auxiliar de enfermagem & 24 & 85,7 \\
\hline Enfermeiro & 4 & 14,3 \\
\hline \multicolumn{3}{|l|}{ Sexo } \\
\hline Feminino & 21 & 75,0 \\
\hline Masculino & 7 & 25,0 \\
\hline \multicolumn{3}{|l|}{ Faixa Etária } \\
\hline $18-25$ & 3 & 10,7 \\
\hline $26-35$ & 12 & 42,9 \\
\hline $36-45$ & 8 & 28,6 \\
\hline 45 ou mais & 4 & 14,3 \\
\hline Não observado & 1 & 3,5 \\
\hline \multicolumn{3}{|l|}{ Turno de trabalho } \\
\hline Manhã & 8 & 28,6 \\
\hline Tarde & 7 & 25,0 \\
\hline Noite & 13 & 46,4 \\
\hline
\end{tabular}

Fonte: Elaboração própria.

Foram observados 235 PVP realizadas pelos profissionais de enfermagem. Na instituição pesquisada, o antisséptico tópico degermante padronizado estava disponível nas pias e bancadas, sendo digluconato de clorexidina $2 \%$, solução degermante, e dispensadores fixados na parede com preparação alcoólica. A higiene das mãos com antisséptico tópico degermante foi realizada em 54 (23,0\%) observações e não foi realizada em 127 (54,0\%). A higienização com solução alcoólica foi efetuada em 25 (10,6\%) observações.

Quanto ao uso de EPI, a máscara descartável foi utilizada em 3 (1,3\%) procedimentos, os óculos de proteção em 5 (2,1\%), o avental em 38 (16,2\%), e a luva de procedimento em 133 $(56,6 \%)$ (Tabela 2$)$.

Tabela 2 - Higiene das mãos com antisséptico tópico degermante, solução alcoólica e adesão às Precauções Padrão por profissionais de enfermagem antes da punção venosa periférica. Uberaba, Minas Gerais, Brasil - 2018. N=235

\begin{tabular}{|c|c|c|}
\hline Variável & $\mathbf{n}$ & $\%$ \\
\hline \multicolumn{3}{|c|}{ Higiene das mãos com antisséptico tópico degermante } \\
\hline $\operatorname{Sim}$ & 54 & 23,0 \\
\hline Não & 127 & 54,00 \\
\hline Não observado & 54 & 23,0 \\
\hline
\end{tabular}


Tabela 2 - Higiene das mãos com antisséptico tópico degermante, solução alcoólica e adesão às Precauções Padrão por profissionais de enfermagem antes da punção venosa periférica. Uberaba, Minas Gerais, Brasil - 2018. N=235

(conclusão)

\begin{tabular}{|c|c|c|}
\hline Variável & $\mathbf{n}$ & $\%$ \\
\hline \multicolumn{3}{|c|}{ Higiene das mãos com solução alcoólica } \\
\hline Sim & 25 & 10,6 \\
\hline Não & 159 & 67,7 \\
\hline Não observado & 51 & 21,7 \\
\hline \multicolumn{3}{|c|}{ Adesão às Precauções Padrão } \\
\hline Máscara descartável & 3 & 1,3 \\
\hline Óculos & 5 & 2,1 \\
\hline Avental & 38 & 16,2 \\
\hline Luvas de Procedimento & 133 & 56,6 \\
\hline
\end{tabular}

Fonte: Elaboração própria.

Dois modelos de CVP estavam em uso no período da coleta de dados, sendo um com dispositivo retrátil (técnica ativa de proteção da agulha) e o outro com proteção da ponta da agulha (técnica passiva de proteção da agulha). O cateter com dispositivo retrátil foi selecionado para uso em $204(86,8 \%)$ procedimentos. O dispositivo de segurança foi acionado após a retirada total da agulha da cânula plástica em 106 (45,1\%); houve contato com sangue em 30 (12,8\%) observações (Tabela 3).

Tabela 3 - Tipo do dispositivo, momento do acionamento do dispositivo ativo de segurança para punção venosa periférica e contato com sangue. Uberaba, Minas Gerais, Brasil -2018. N=235

\begin{tabular}{|c|c|c|}
\hline Variável & $\mathbf{n}$ & $\%$ \\
\hline \multicolumn{3}{|l|}{ Tipo do dispositivo } \\
\hline Dispositivo retrátil e técnica ativa de proteção da agulha & 204 & 86,8 \\
\hline $\begin{array}{l}\text { Dispositivo de proteção da ponta da agulha e técnica passiva de } \\
\text { proteção }\end{array}$ & 28 & 11,9 \\
\hline Não observado & 3 & 1,3 \\
\hline \multicolumn{3}{|l|}{ Momento para acionamento do dispositivo de segurança } \\
\hline Logo após a visualização do refluxo sanguíneo & 56 & 23,8 \\
\hline $\begin{array}{l}\text { Durante a retirada da agulha e inserção da cânula plástica } \\
\text { (simultâneo) }\end{array}$ & 69 & 29,4 \\
\hline Após a retirada total da agulha da cânula plástica & 106 & 45,1 \\
\hline Não observado & 4 & 1,7 \\
\hline \multicolumn{3}{|l|}{ Contato com sangue } \\
\hline Sim & 30 & 12,8 \\
\hline Não & 202 & 86,0 \\
\hline Não observado & 3 & 1,3 \\
\hline
\end{tabular}

Fonte: Elaboração própria.

Após o procedimento de PVP, o perfurocortante foi transportado da enfermaria (leito) até o expurgo ou posto de enfermagem para descarte da agulha em caixa coletora para material perfurocortante, uma vez que não estava disponível beira leito. O transporte do perfurocortante e outros resíduos foi realizado em bandeja plástica e pelas mãos dos profissionais sem embalagem em $170(72,3 \%)$ e $31(13,2 \%)$ procedimentos, respectivamente, e diretamente pelas mãos dos profissionais em $24(10,2 \%)$ procedimentos na própria embalagem (Tabela 4). 
Tabela 4 - Transporte do cateter venoso periférico e descarte do dispositivo logo após o procedimento. Uberaba, Minas Gerais, Brasil -2018. N=235

\begin{tabular}{|c|c|c|}
\hline Variável & $\mathbf{n}$ & $\%$ \\
\hline \multicolumn{3}{|l|}{ Transporte do cateter venoso periférico } \\
\hline Bandeja plástica & 170 & 72,3 \\
\hline Mãos dos profissionais sem embalagem & 31 & 13,2 \\
\hline Mãos dos profissionais com embalagem & 24 & 10,2 \\
\hline Não observado & 10 & 4,3 \\
\hline \multicolumn{3}{|c|}{ Descarte do dispositivo logo após o procedimento } \\
\hline Sim & 219 & 93,2 \\
\hline Não & 12 & 5,1 \\
\hline Não observado & 4 & 1,7 \\
\hline
\end{tabular}

Fonte: Elaboração própria.

\section{Discussão}

A amostra constitui-se por 28 profissionais de enfermagem, com predominância de indivíduos do sexo feminino (75\%), como mostrado em outros estudos sobre a temática ${ }^{(16)}$. Trata-se de uma característica histórica da enfermagem, profissão exercida nos seus primórdios apenas por mulheres $^{(17)}$.

A adesão de técnicos e auxiliares de enfermagem $(85,6 \%)$ foram maiores. Esse dado vai ao encontro com a literatura brasileira em virtude de esta ser a classe de maior número dentre os profissionais de enfermagem, e por prestar assistência direta e constante aos pacientes, realiza mais procedimentos ${ }^{(18-19)}$.

As PP são indicadas e devem ser aplicadas a qualquer paciente, independentemente do diagnóstico clínico, sendo recomendados máscara descartável, óculos, avental e luvas de procedimento, sempre que houver contato com sangue e fluidos ${ }^{(20)}$. No presente estudo foi mostrado que em 133 (56,6\%) procedimentos os profissionais não utilizaram luvas de procedimento para a realização da punção. Estudo brasileiro também apontou que os profissionais não usaram luvas de procedimento na PVP; os profissionais justificaram nessa investigação perda do tato no momento da palpação da veia e da punção ${ }^{(21)}$.

A higienização das mãos antes da realização do procedimento de PVP foi baixa (54\%). Este é um fator preocupante, visto que a não higienização está diretamente relacionada às infecções relacionadas à assistência (IRAS) ${ }^{(22)}$. A baixa adesão à higiene das mãos antes do procedimento invasivo, incluindo PVP, também foi apontada em outros estudos ${ }^{(23-24)}$. Mesmo com o reconhecimento de sua importância, a não adesão dos profissionais de saúde em realizar frequentemente essa prática é um problema mundialmente discutido ${ }^{(25)}$.

Para escolha do modelo do dispositivo, o profissional deve considerar a segurança do paciente, a rede venosa do paciente e o tipo de indicação da PVP, para escolha adequada do cateter $^{(26)}$. Nesta investigação, o modelo do dispositivo mais utilizado foi o de técnica ativa de acionamento, visto a maior disponibilidade do modelo no hospital em estudo.

A maioria dos profissionais utilizaram o dispositivo de modo incorreto, acionando o mecanismo de proteção retrátil somente após a retirada total da agulha da veia, o que pode contribuir para o risco de picada de agulha e, ainda, respingo de sangue em mucosas, pele íntegra e não íntegra ${ }^{(27)}$.

Estudo que comparou a eficácia entre os DSCE passivo e ativo de segurança demonstrou que o modelo de acionamento passivo foi relacionado às menores taxas de acidentes com perfurocortantes ${ }^{(28)}$. Em contrapartida, em outro estudo, o DSCE houve aumento de 30\% nos acidentes e o custo aumentou 211,08\% em gastos para o hospital $^{(29)}$.

Em estudo americano, dentre os 110 acidentes ocorridos com CVP com dispositivo de segurança ativo, em 42 destes o dispositivo não foi acionado $^{(30)}$. A maioria dos acidentes causados por agulha com DSCE pode ser evitada pela capacitação dos profissionais, estimulação 
do descarte seguro da agulha e pelo uso adequado do dispositivo de acordo com as indicações do fabricante ${ }^{(31)}$.

Durante as observações foi visto que houve o contato com sangue $(\mathrm{n}=30)$ principalmente envolvendo técnicos e auxiliares, por estes realizarem mais PVP que o enfermeiro ${ }^{(32)}$. A exposição ao sangue mostra um grande risco à saúde do profissional, evidenciando a possibilidade de contaminação para várias doenças, como hepatite B, HIV entre outras ${ }^{(33)}$.

O descarte do dispositivo em caixas específicas para perfurocortantes foi realizado em grande parte das observações, no entanto, o transporte foi, muitas vezes, realizado de forma inadequada, pelas mãos dos profissionais sem embalagem, o que os expõe ao risco biológico. Nesse aspecto, o descarte e o uso correto do dispositivo contribuem para a diminuição dos números de acidentes, como mostrado em estudo realizado em São Luís do Maranhão ${ }^{(34)}$.

A utilização de DSCE e a adesão às precauções padrão podem contribuir para maior conforto ao paciente e segurança aos profissionais, porém não depende exclusivamente da adoção de novos dispositivos, mas sim, de conscientização e atitudes preventivas pela equipe de enfermagem.

Este estudo apresenta algumas limitações, pois não foi possível avaliar os procedimentos realizados simultaneamente e ter sido realizado em uma única unidade assistencial, o que dificulta generalizações.

\section{Conclusão}

A maioria dos profissionais de enfermagem utilizaram o CVP com controle de engenharia de modo incorreto, acionando o sistema de proteção retrátil após a retirada total da agulha da veia. A adesão dos profissionais de enfermagem às PP foi baixa.

Ressalta-se a importância da realização de educação continuada com esses profissionais, sendo necessário rever as ações de proteção à saúde do trabalhador, de modo a aumentar a adesão às PP e ao uso correto do dispositivo de segurança com controle de engenharia. Espera-se que os resultados encontrados nesta pesquisa possam contribuir para a adesão dos profissionais às práticas seguras, promovendo estratégias para melhoria do cuidado prestado.

\section{Fontes de financiamento}

Fundação de Amparo à Pesquisa do Estado de Minas Gerais (FAPEMIG). Processo número 00146/2016.

\section{Colaborações:}

1 - concepção, projeto, análise e interpretação dos dados: Guilherme Nascimento de Azevedo, Letícia Nunes Torres, Lúbia Alves dos Santos e Silmara Elaine Malaguti Toffano;

2 - redação do artigo e revisão crítica relevante do conteúdo intelectual: Damiana Aparecida Trindade Monteiro e Elucir Gir;

3 - aprovação final da versão a ser publicada: Silmara Elaine Malaguti Toffano.

\section{Referências}

1. Phillips LD, Gorski LA. Manual of I.V. Therapeutics: Evidence-Based Practice for Infusion Therapy. 6th ed. Philadelphia (US): Davis Company; 2013.

2. Zerati AE, Wolosker N, Luccia N, Puech-Leão P. Cateteres venosos totalmente implantáveis: histórico, técnica de implante e complicações. J Vasc Bras. 2017;16(2):128-39. DOI: http://dx.doi. org/10.1590/1677-5449.008216

3. Souza-Junior VD, Mendes IAC, Marchi-Alves LM, Jackman D, Wilson-Keates B, Godoy S. Peripheral Venipuncture Education Strategies for Nursing Students: An Integrative Literature Review. J Infus Nurs. 2020;43(1):24-32. DOI:10.1097/nan. 0000000000000351

4. López JLG, Vilela AA, Palacio EF, Corral JO, Martí CB, Portal PH. Indwell times, complications and costs of open vs closed safety peripheral intravenous catheters: a randomized study. J Hosp Infect. 2014;86(2):117-26. DOI: https://doi. org/10.1016/j.jhin.2013.10.008

5. Barros DX, Tipple AFV, Lima LKOL, Souza ACS, Neves ZCP, Salgado TA. Análise de 10 anos de 
acidentes com material biológico entre a equipe de enfermagem. Rev eletrônica enferm. 2016;18: 1-10. DOI: https://doi.org/10.5216/ree.v18.35493

6. Silva SMS, Gomes IVMPS, Anjos MS. Acidente perfurocortante: conhecimento e uso de dispositivos de segurança. Rev Saúde. com [Internet]. 2016 [cited 2021 Mar 7];12(2):522-7. Available from: http://periodicos2.uesb.br/index. $\mathrm{php} / \mathrm{rsc} /$ article/view/406

7. Barbosa ASAA, Diogo GA, Salotti SRA, Silva SMUR. Subnotificação de acidente ocupacional com materiais biológicos entre profissionais de Enfermagem em um hospital público. Rev Bras Med Trab. 2017;15(1):12-7. DOI: http://dx.doi.org/10.5327/Z1679443520177034

8. Ferreira LA, Peixoto CA, Paiva L, Silva QCG, Rezende MP, Barbosa MH. Adesão às precauções padrão em um hospital de ensino. Rev Bras Enferm. 2017;70(1):96-103. DOI: http://dx.doi. org/10.1590/0034-7167-2016-0138

9. Santos LT, Rocha FLR, Marziale MHP. Agulhas com dispositivos de segurança e a prevenção de acidentes: revisão integrativa. Rev Bras Enferm. 2018;71(6):3084-92. DOI: http://dx.doi. org/10.1590/0034-7167-2017-0719

10. Stringer B, Astrakianakis G, Haines T, Kamsteeg K, Danyluk Q, Tang T, et al. Conventional and sharp safety devices in 6 hospitals in British Columbia, Canada. Am J Infect Control. 2011;39(9):738-45. DOI: http://dx.doi.org/ 10.1016/j.ajic.2010.12.004

11. Ottino MC, Argentero A, Argentero PA, Garzaro G, Zotti CM. Needlestick prevention devices: data from hospital surveillance in Piedmont, Italy-comprehensive analysis on needlestick injuries between healthcare workers after the introduction of safety devices. BMJ Open. 2019;9:e030576. DOI: 10.1136/bmjopen-2019030576

12. Roff M, Basu S, Adisesh A. Do active safety-needle devices cause spatter contamination? J Hosp Infect. 2014;(86):221-3. DOI: https://doi. org/10.1016/j.jhin.2014.01.006

13. Mitchell AH, Parker GB, Kanamori H, Rutala WA, Weber DJ. Comparing non-safety with safety device sharps injury incidence data from two different occupational surveillance systems. J Hosp Infect. 2017;(96):195-8. DOI: https://doi.org/10.1016/j.jhin.2017.02.021
14. Silva GF, Rocha DO, Capelete AIGB, Silva CP. Subnotificações de acidentes de trabalho com material biológico de profissionais da enfermagem de um hospital do Paraná. Var Sci - Ci Saúde. 2020;6(2). DOI: https://doi.org/10.48075/vscs. v6i2.26238

15. Reddy VK, Lavoie MC, Verbeek JH, Pahwa M. Devices for preventing percutaneous exposure injuries caused by needles in healthcare personnel. CochraneDatabaseSystRev. 2017;11(11):CD009740. DOI:10.1002/14651858.CD009740.pub3

16. Silveira RCP, Ribeiro IKS, Mininel VA. Calidad de vida, perfil sociodemográfico y laboral del personal de enfermería de un hospital universitário. Enferm actual Costa Rica. 2021:41. DOI: 10.15517/ revenf.v0i41.44769

17. Melo EM, Aragão AL, Pessoa CMP, Lima FET, Barbosa IV, Studart RMB, et al. Cuidados dispensados pela equipe de enfermagem durante o procedimento de punção venosa periférica. Rev Enferm UFPE. 2015;9(3). DOI: 10.5205/ reuol.7505-65182-1-RV.0903201502

18. Arantes MC, Haddad MCFL, Marcon SS, Rossaneis MA, Pissinati PSC, Oliveira SA. Acidentes de trabalho com material biológico em trabalhadores de serviços de saúde. Cogitare enferm. 2017;(22)1. DOI: http://dx.doi. org/10.5380/ce.v22i1.46508

19. Souza LS. Clima organizacional e ocorrência de acidentes com materiais perfurocortantes num hospital público do Estado de São Paulo [dissertação]. Ribeirão Preto (SP): Universidade de São Paulo, Escola de Enfermagem de Ribeirão Preto; 2016. DOI:10.11606/D.22.2017. tde-28032017-153644

20. Siegel JD, Rhinehart E, Jackson M, Chiarello L. Guidelines for Isolation Precautions: Preventing Transmission of Infectious Agents in Health Care Settings. Am J Infect Control. 2007;35(10 Suppl 2):S65-S164. DOI: https://doi.org/10.1016/j. ajic.2007.10.007

21. Batista OMA, Moreira RF, Sousa AFL, Moura MEB, Andrade D, Madeira MZA. Complicações locais da terapia intravenosa periférica e fatores associados em hospital brasileiro. Rev Cubana Enferm [Internet]. 2018 [cited 14 Jul 2021];34(3). Available from: http:// www.revenfermeria.sld.cu/index.php/enf/article/ view/1246 
22. Oliveira HM, Silva CPR, Lacerda RA. Policies for control and prevention of infections related to healthcare assistance in Brazil: a conceptual analysis. Rev esc enferm USP. 2016;50(3):505-11. DOI: https://doi.org/10.1590/ S0080-623420160000400018

23. Oliveira AC, Pinto SA. Participação do paciente na higienização das mãos entre profissionais de saúde. Rev Bras Enferm. 2018;71(2):259-64. DOI: https://doi.org/10.1590/0034-7167-2016-0124

24. Medrado MMPM, Correa I. Processo de observação da lavagem das mãos em relação ao tipo de procedimento realizado. Rev UNINGÁ [Internet]. 2017 [cited 2019 Mar 7];35(1). Available from: http://revista.uninga.br/index.php/uninga/ article/view/1079

25. Giordani AT, Sonobe HM, Ezaias GM, Valério MA, Andrade D. Adesão da equipe de enfermagem à higienização das mãos: fatores motivacionais. Rev Rene. 2014;15(4):559-68. DOI: https://doi.org/10.15253/ 2175-6783.2014000400002

26. Oliveira AM, Danski MTR, Pedrolo E. Punção venosa periférica guiada por ultrassonografia: prevalência de sucesso e fatores associados. Cogitare enferm. 2017;22(3). DOI: http://dx.doi. org/10.5380/ce.v22i3.49599

27. Ansari A, Ramaiah P, Collazo L, Salihu HM, Haiduven D. Comparison of visual versus microscopic methods to detect blood splatter from an intravascular catheter with engineered sharps injury protection. Infect Control Hosp Epidemiol. 2013;34(11)1174-80. DOI: https://doi. org/10.1086/673462

28. Tosini W, Ciotti C, Goyer F, Lolom I, L'Hériteau F, Abiteboul D, et al. Needlestick Injury Rates According to Different Types of Safety-Engineered Devices: Results of a French Multicenter Study. Infect Control Hosp
Epidemiol. 2010;31(4):402-7. DOI: https://doi. org/10.1086/651301

29. Mendes RNC, Silva LGC, Haddad MCL, Moreno FN, Gil RB. Custo-efetividade de agulhas e cateteres sobre agulha com dispositivos de proteção ativa no contexto hospitalar. Texto contexto - enferm. 2015;24(3):867-74. DOI: https:// doi.org/10.1590/0104-07072015002870014

30. Green-Mckenzie J, McCarthy RB, Shofer FS. Characterisation of occupational blood and body fluid exposures beyond the Needlestick Safety and Prevention. J Infect Prev. 2016;17(5):226-32. DOI: https://doi.org/10.1177/1757177416645339

31. Schuurmans J, Lutgens SP, Groen L, Schneeberger PM. Do safety engineered devices reduce needlestick injuries? J Hosp Infect. 2018;100(1):99104. DOI: https://doi.org/10.1016/j.jhin.2018.04.026

32. Carvalho DC, Rocha JG, Gimenes MCA, Santos EC, Valim MD. Acidentes de trabalho com material biológico na equipe de enfermagem de um hospital do Centro-Oeste brasileiro. Esc Anna Nery. 2018;22(1):e20170140. DOI: http://dx.doi. org/10.1590/2177-9465-ean-2017-0140

33. Maristela AMM, Jairo AA. Acidentes com material biológico: a realidade de uma instituição hospitalar do interior paulista. Rev Min Enferm [Internet]. 2009 [cited 2021 Mar 7];13(1):115-22. Available from: https://cdn.publisher.gn1.link/reme.org.br/ pdf/v13n1a17.pdf

34. Santos SR, Novaes CO. Profile of accidents with sharps among health professionals from a hospital of the public network at São Luís city. R pesq cuid fundam online. 2018;10(4):977-85. DOI: http:// dx.doi.org/10.9789/2175-5361.2018.v10i4.977-985

Recebido: 5 de agosto de 2021

Aprovado: 7 de novembro de 2021

Publicado: 22 de novembro de 2021

A Revista Baiana de Enfermagem utiliza a Licença Creative Commons - Atribuição-NãoComercial 4.0 Internacional. https://creativecommons.org/licenses/by-nc/4.0/

Este artigo é de acesso aberto distribuído sob os termos da Licença Creative Commons (CC BY-NC). Esta licença permite que outros remixem, adaptem e criem a partir do seu trabalho para fins não comerciais. Embora os novos trabalhos tenham de lhe atribuir o devido crédito e não possam ser usados para fins comerciais, os usuários não têm de licenciar esses trabalhos derivados sob os mesmos termos. 